\title{
25 Research Soure \\ Identification of the First C1 Subgenotype of \\ Enterovirus 71 in Mainland of China in a \\ Retrospective Study
}

\section{Fenfen SI}

National Institute for Viral Disease Control and Prevention

\section{Dongyan WANG}

National Institute for Viral Disease Control and Prevention

Tianjiao Jl

National Institute for Viral Disease Control and Prevention

\section{Yong ZHANG}

National Institute for Viral Disease Control and Prevention

\section{Shuangli ZHU}

National Institute for Viral Disease Control and Prevention

Junhan LI

National Institute for Viral Disease Control and Prevention

\section{Wenbo XU}

National Institute for Viral Disease Control and Prevention

\section{Zexin TAO}

Shandong Center for Disease Control and Prevention

Dongmei YAN ( $\nabla$ dongmeiyan1976@163.com )

National Institute for Viral Disease Control and Prevention

\section{Short report}

Keywords: C1 sub-genotype of Enterovirus 71Keywords,Retrospective study, Guillain-Barre syndrome, Acute Flaccid Paralysis.

Posted Date: January 12th, 2022

DOI: https://doi.org/10.21203/rs.3.rs-421089/v2

License: (c) (1) This work is licensed under a Creative Commons Attribution 4.0 International License. Read Full License 


\section{Abstract}

The C4 sub-genotype of Enterovirus 71(EV71) is identified as the most dominant sub-genotype circulating in Chinese mainland since 1998, while the circulation situation of EV71 before 1998 is not well established due to insufficient experimental data. The C1 sub-genotype of EV71 has not been reported in Chinese mainland by now. Based on AFP surveillance system of the mainland of China, this study performed a retrospective study of AFP cases for 1985-1999ロA strain of EV-A71 C1 sub-genotype was found. To our knowledge, this strain (SD92-41) is the first C1 sub-genotype reported in Chinese mainland. This study proves that the $\mathrm{C} 1$ gene subtype also appeared in Chinese mainland, but it is not clear whether it is an imported or a local epidemic strain. With sufficient information from retrospective studies, the source of the SD92-41 strain will be identified, and the prevalence of EV-A71 in Chinese mainland before 1998 will be clearer.

\section{Introduction}

As a member of the human Enterovirus species A (genus Enterovirus, family Picornaviridae), Enterovirus 71 (EV71) is a small, non-enveloped, positive-stranded RNA virus. Based on the VP1 coding region, the worldwide circulation of EV71 can be divided into seven genotypes designated A to G, where the two major genotypes $B$ and $C$ are classified into 14 subgenotypes respectively designated $B 0$ to $B 7$ and $C 1$ to C6(1). The sub-genotype $\mathrm{C} 4$ has been identified as the most dominant sub-genotype circulating in Chinese mainland since 1998, however the circulation situation of EV71 before 1998 is not well established due to insufficient experimental data(2). It is reported that the sub-genotype C1 of EV71 circulated only in the Western Pacific, Europe and the United States before 2000. After 2000, it was reported that $\mathrm{C} 1$ genotype was prevalent in Southeast Asia, such as Malaysia, Thailand, Hong Kong, however the sub-genotype C1 of EV71 has not been reported in Chinese mainland by now(3). China established AFP (Acute Flaccid Paralysis, AFP) surveillance system to monitor cases that acute onset, decreased muscle tension, decreased muscle strength and weakened or disappeared tendon reflex. Based on AFP surveillance system of the mainland of China, this study performed a retrospective study of AFP cases for 1985-1999: A strain of EV-A71 C1 sub-genotype was found.

\section{Material And Methods}

172 human rhabdomyosarcoma (RD) cell positive virus isolates from AFP surveillance system in Chinese mainland from 1985 to 1999 were retrospectively studied and molecular typing method was performed. Primer pair $Y 7 / Q 8$ was used for poliovirus screening, and for the isolates with negative results, primer pairs 486/488 and 040/011 were used to amplify the partial VP1 sequences and the combination of the two sequences yielded the entire VP1 coding region. 164 polioviruses and 8 (4.7\%) NPEV strains were identified. 8 NPEVs included 2 Coxsackievirus B5, 2 Echovirus 1, 1 EV71, 1 Echovirus 26, 1 Coxsackievirus B1 and 1 Enterovirus-B.

\section{Results}


The EV71 strain (named SD92-41) was isolated from a 7-year-old male patient with AFP in Shandong Province in 1992. The AFP case was diagnosed as Guillain-Barre syndrome (GBS) by a polio diagnosis expert panel, and the patient had no residual paralysis during the 60-day clinical follow-up. Phylogenetic analysis based on entire VP1 coding regions of EV71 was conducted with the maximum-likelihood (ML) method revealed that the SD92-41 strain belonged to the C1 sub-genotype(Fig. 1). The VP1 genome sequence of the SD92-41 strain and the EV-71 prototype strain showed $96.9 \%$ similarity in nucleotide sequence and $80.8 \%$ similarity in amino acid sequence. Homologous comparison revealed that the VP1 genome sequence of the SD92-41 strain had the highest homology with the strain 9718-TX-89 from U.S. in 1989 and had $99.7 \%$ similarity in nucleotide sequence and $96.2 \%$ similarity in amino acid sequence.

The whole genome sequence of the SD92-41 strain was determined to be $7411 \mathrm{nt}$ long. The ORF of the SD92-41 strain is $6582 \mathrm{nt}$ in length, encoding a polypeptide of 2193 amino acids, with a 746nt 5'-UTR and a 83nt 3'-UTR. Phylogenetic trees based on VP1, P1, P2, P3 coding regions of prototypes strains of the human enterovirus species A and EV71 typical strains were constructed with the maximum-likelihood (ML) method (Fig. 2). The topology of the phylogenetic tree based on the nucleotide sequences of the P1 region was similar to that of the VP1 region,the SD92-41 strain and other sub-genotypes of EV71 were clustered into a large cluster (Fig. 2a and Fig. 2b). However, SD92-41 and CVA8 were clustered into one branch in P2 and P3 regions, indicating that SD92-41 and CVA8 may be recombined. Simplot analysis showed that SD92-41 and CVA8 had highly gene recombination in P2 and P3 regions (Fig. 3). It's worth noting that SD92-41 and the other two EV71 strains (480/Netherlands/1991-AB115492 and 5746/Taiwan/1998-AF304457) were clustered in the same branch in P2 and P3 regions. This means that two EV71 strains have high similarity to SD92-41 in the P2 and P3 regions.

\section{Discussion}

Since 1998, the C4 subgenotype strains of Enterovirus 71 have been persistently circulating in Chinese mainland for 22 Years(2), where only one or two imported subgenotypes were reported(4). Reports on the circulation situation of EV71 before 1998 are few. One sequence from Heilongjiang in 1997 and one sequence from ShanDong in 1996 were identified as C3 and C2 subgenotype respectively, indicating that the C3 and C2 subgenotypes appeared in mainland of China(5)(6). To our knowledge, SD92-41 is the first C1 subgenotype reported in Chinese mainland, which showed high sequence similarity in VP1 region to the strain 9718-TX-89 from U.S. in 1989, but there is no direct evidence supporting the association with the American strain. SD92-41 has a sequence homology in the whole genome region to the two other EV71 strains (480/Netherlands/1991-AB115492 and 5746/Taiwan/1998-AF304457), we inferred from the experimental fact that the recombination events might occur first in the common ancestor and then they spread to different countries. This study proves that the $\mathrm{C} 1$ gene subtype also appeared in Chinese mainland, but the source of the SD92-41 strain is not clear because of insufficient information.

\section{Abbreviations}


EV71

Enterovirus 71

AFP

Acute Flaccid Paralysis

RD

rhabdomyosarcoma

GBS

Guillain-Barre syndrome

\section{Declarations}

\section{Ethics approval and consent to participate:}

Animal and Human Rights Statement This study did not involve human experimentation. Biosafety evaluations were approved by the National Institute for Viral Disease Control and Prevention, China CDC. All experimental operations were handled following the Standard Operational Protocol approved by China CDC.

\section{Consent for publication}

Written informed consent for specimen collection, testing and publication was obtained from all individuals (or their parents) in the study population.

\section{Availability of data and materials}

Condensed anonymized data are available from the corresponding author on reasonable request. Whole genome nucleotide sequences for the strain determined in this study have been deposited in the GenBank nucleotide sequence database under accession numbers MW473684.

\section{Competing interests $\square$}

The authors declare no competing interests.

\section{Author' Contributions [}

Conceptualization, Fenfen Si, Dongmei Yan; formal analysis, Fenfen Si, Dongyan Wang, Tianjiao Ji, Yong Zhang, Shuangli Zhu, Junhan Li, Wenbo Xu, Dongmei Yan; Writing-original draft preparation, Fenfen Si; Writing-review and editing, Dongmei Yan; Project administration,Dongmei Yan; funding acquisition, Dongmei Yan. All authors have read and agreed to the published version of the manuscript.

\section{Funding:}

This study was supported by the National Key Technology R\&D Program of China (Project No. 2017ZX10104001). We also acknowledge the funding received from the Key Technologies R\&D Program 
of the National Ministry of Science (Project Nos. 2018ZX10101002-004-006) $\square$ Taishan Scholar Program for Young Experts (tsqn202103187) $\square$ National key R \& D plan (2021YFC0863000) and Municipal Natural Science Foundation of Beijing of China (L192014). The funding body was not involved in the study design, clinical sample collection, data analysis, and interpretation or writing of the manuscript.

\section{Acknowledgements:}

We thank the National Institute for Viral Disease Control and Prevention, for the unidentifed isolate from AFP surveillance.

\section{References}

1. Chang P-C, Chen S-C, Chen K-T. The Current Status of the Disease Caused by Enterovirus 71 Infections: Epidemiology, Pathogenesis, Molecular Epidemiology, and Vaccine Development. IJERPH. 2016 Sep 9;13(9):890.

2. Zhang $Y$, Wang J, Guo W, Wang H, Zhu S, Wang D, et al. Emergence and transmission pathways of rapidly evolving evolutionary branch $\mathrm{C} 4 \mathrm{a}$ strains of human enterovirus 71 in the Central Plain of China. PLoS One. 2011;6(11):e27895.

3. Liu Z, Gui J, Hua Q, Dong C. Molecular epidemiology and evolution of human enterovirus 71 and hand, foot and mouth disease. Yi Chuan. 2015 May;37(5):426-35.

4. Liu Y, Zhang F, Fu C, Wu S, Chen X, Shi Y, et al. Combination of intratypic and intertypic recombinant events in EV71: a novel evidence for the "triple-recombinant" strains of genotype A viruses in Mainland China from 2008 to 2010. Virus Genes. 2015 Jun;50(3):365-74.

5. Shimizu H, Utama A, Onnimala N, Li C, Li-Bi Z, Yu-Jie M, et al. Molecular epidemiology of enterovirus 71 infection in the Western Pacific Region. Pediatr Int. 2004 Apr;46(2):231-5.

6. Tao Z, Wang H, Xu A. Identification of a C2 Subgenogroup Strain of Enterovirus 71 in a Retrospective Study in Shandong Province, China, from 1990 to 2010. Journal of Clinical Microbiology. 2012 May 1;50(5):1823-4.

\section{Figures}




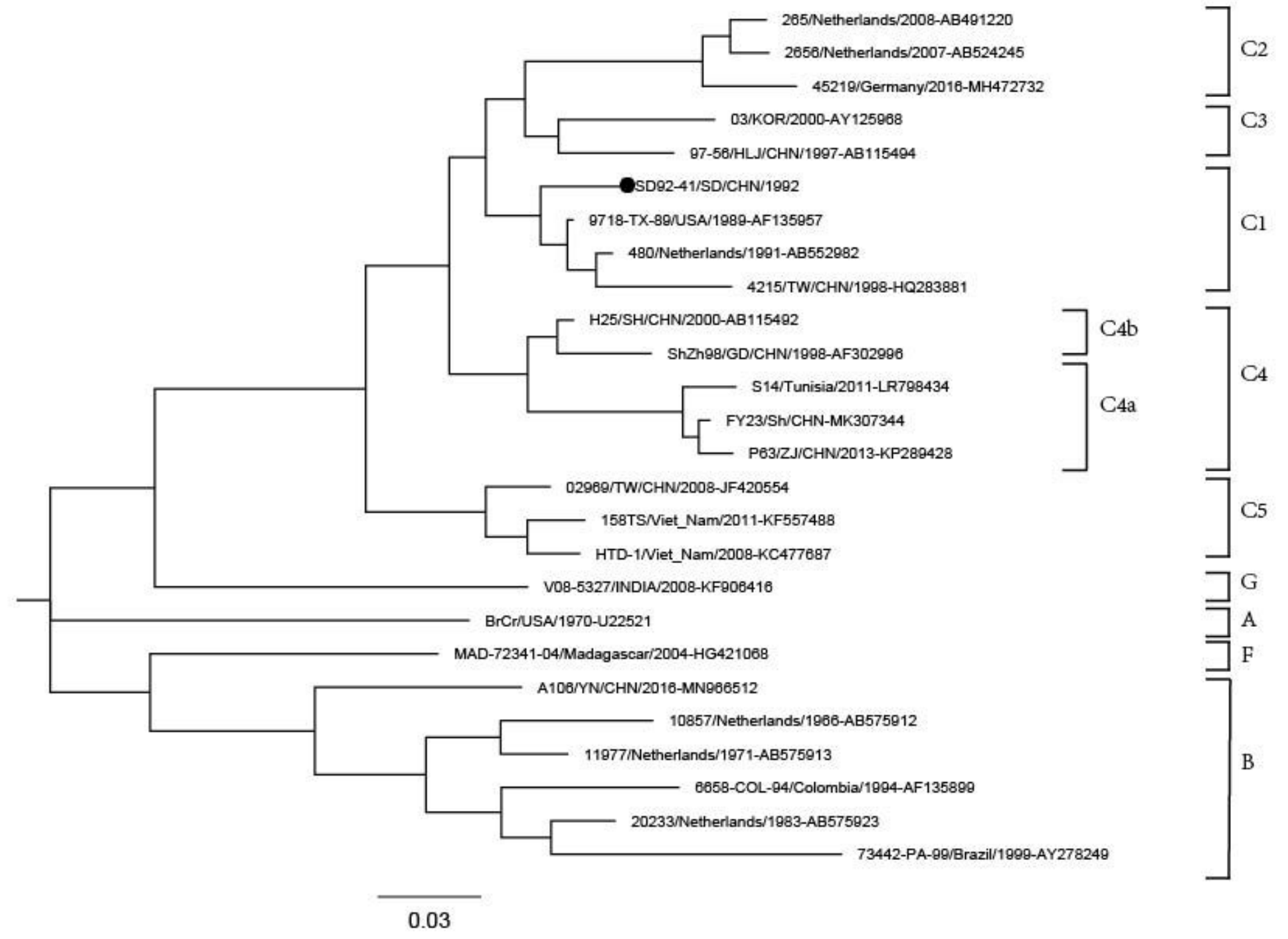

\section{Figure 1}

Phylogenetic Tree Based on Entire VP1 Coding Regions of EV71.

Note: The SD92-41 strain is marked with a black solid circular. 

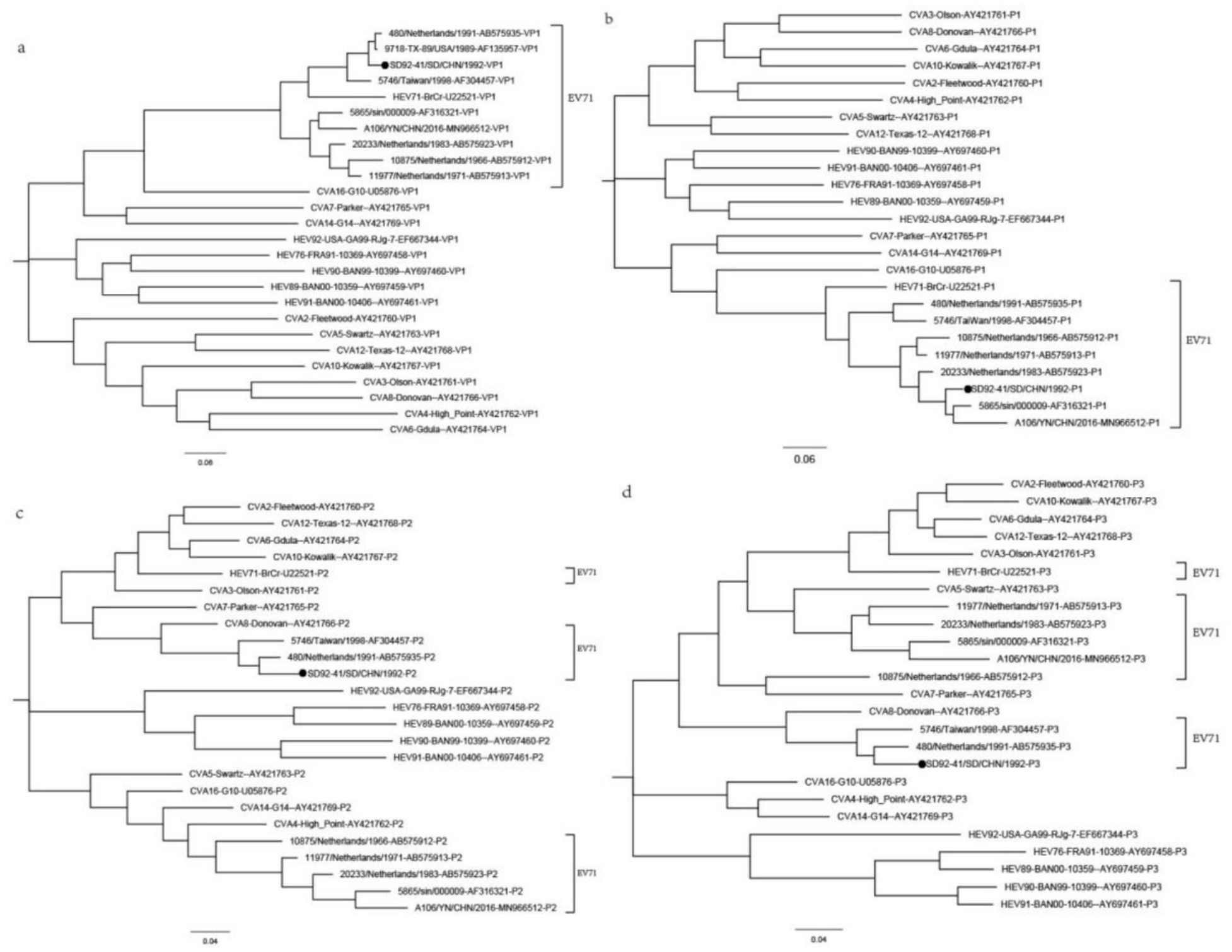

Figure 2

Phylogenetic Tree Based on the VP1, P1, P2, and P3 sequences of EV-A.

Note: Maximum likelihood trees were constructed using the GTR + G model and were implemented in MEGA7.0 with 1000 bootstrap replicates. The close circle represents the SD92-41 strain. The scale bars indicate the genetic distance. (a) VP1 coding sequence; (b) P1 coding sequence; (c) P2 coding sequence; (d) P3 coding sequence. 

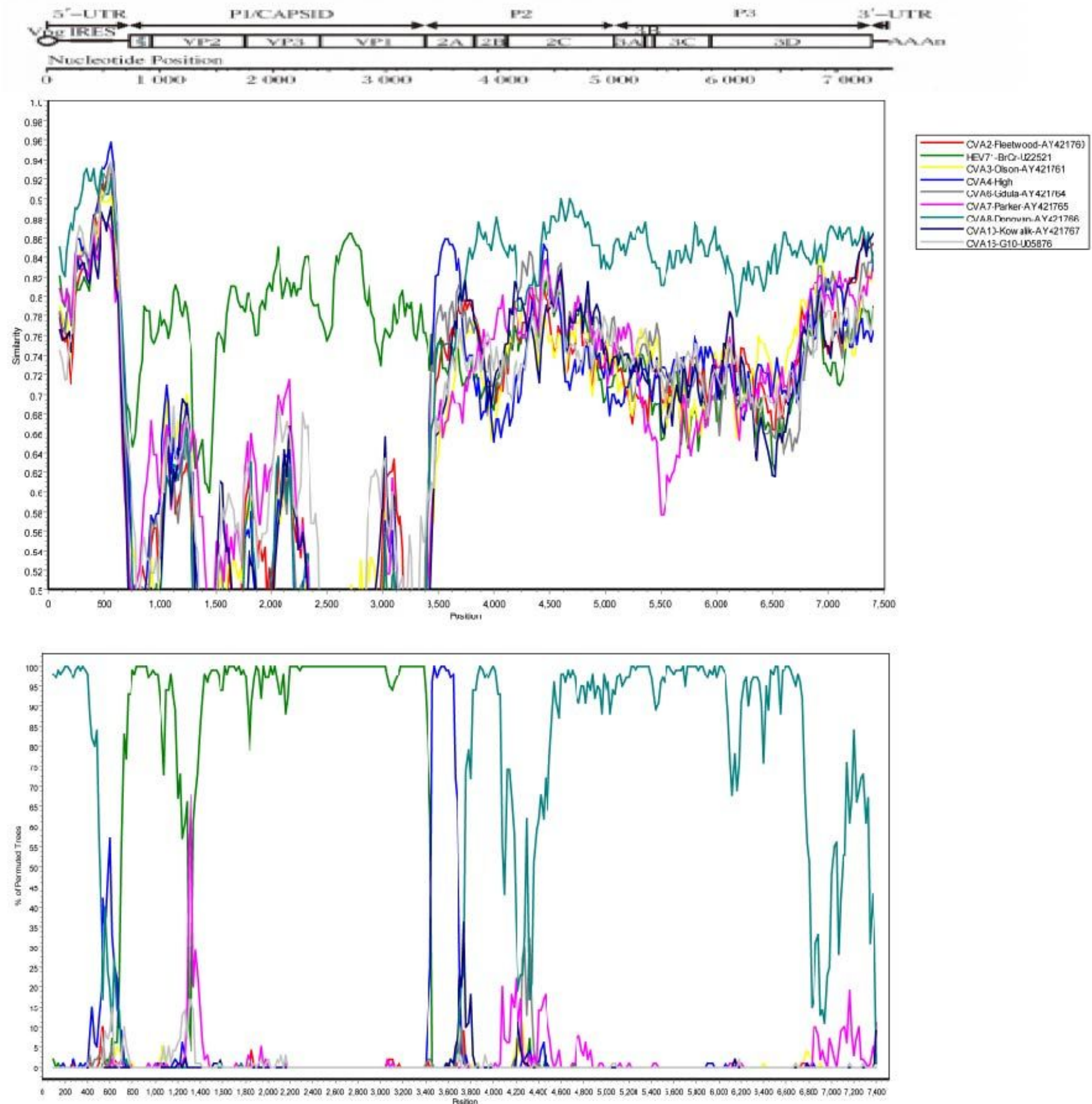

Figure 3

Similarity and Bootscanning Ananlysis of the SD92-41 Strain and Other EV-A Strains.

The SD92-41 strain was used as a query sequence. 